\title{
UTILIZAÇÃO DE ENXERTO AUTÓGENO DE CRISTA ILÍACA PÓS RESSECÇÃO DE AMELOBLASTOMA EM MANDÍBULA: RELATO DE CASOS CLÍNICOS
}

\author{
AUTOGENOUS ILIAC CREST GRAFTING AFTER JÁW'S AMELOBLASTOMA \\ RESSECTION: REPORT OF CLINICAL CASES
}

\section{INDARA DE MELO SOUZA}

Graduanda na Faculdade de Odontologia da Universidade Federal Fluminense, Niterói/RJ.

\section{ALEXANDRE M. DE MORAES}

Cirurgião Bucomaxilofacial do Hospital Municipal Salgado Filho, Rio de Janeiro/ RJ.

\section{${ }^{3}$ ADRIANA TEREZINHA NOVELINO}

Professora da Disciplina de Clínica Diagnóstica da Universidade Federal Fluminense, Niterói/RJ.

\section{${ }^{4}$ MARCELO JOSÉ UZEDA}

Professor das Disciplinas de Cirurgia Oral Menor e Anestesiologia da Universidade Federal Fluminense, Niterói/RJ.

\section{${ }^{5}$ RODRIGO BRITO FIGUEIREDO RESENDE}

Professor das Disciplinas de Cirurgia Oral Menor e Anestesiologia da Universidade Federal Fluminense, Niterói/RJ.

Universidade Federal Fluminense

Faculdade de Odontologia

\section{Indara de Melo Souza}

Rua Mario Santos Braga, número 28- Niterói/ RJ 24020-140

Cel: (21) 997575898.

E-mail: indaramelo@hotmail.com. 


\title{
RESUMO
}

O ameloblastoma é o segundo tumor odontogênico benigno mais comum descrito na literatura, com perfil de crescimento lento, infiltrativo e agressivo. Apresenta predileção pela região posterior da mandíbula, sendo por vezes assintomático, podendo atingir grandes proporções. Sua origem advém do epitélio odontogênico e o diagnóstico deve ser realizado por meio de exames de imagem e uma biópsia incisional para exame histopatológico. O tratamento é cirúrgico, podendo-se utilizar técnicas conservadoras ou radicais. Para as ressecções totais ou parciais, deve ser planejada a reabilitação do paciente, devolvendo assim a função e a estética. O osso autógeno, é considerado o "padrão ouro" quando se opta por enxertia nesse tipo de lesão. O objetivo deste trabalho é descrever dois casos cujo tratamento consistiu em mandibulectomia parcial e enxertia de osso autógeno de crista ilíaca não vascularizado, comparando as evoluções por um período de um ano após as intervenções, realizadas no Hospital Municipal Salgado Filho (HMSF) Rio de Janeiro/RJ. Pôde-se observar que embora o tratamento de eleição tenha sido o mesmo para ambos os casos, os resultados finais foram distintos entre os pacientes.

Palavras Chave: Ameloblastoma, Tumores Odontogênicos, Enxerto Autógeno

\begin{abstract}
Ameloblastoma is the second most common benign odontogenic tumor describe in the literature with a slow, infiltraive and aggressive growth profile. It shows predilection for the posterior jaw's region, being sometimes asymptomatic causing it reach large proportions. Its comes from the odontogenic epithelium and the diagnosis must be made by trough imaging exams and incisional biopsy for histophatological examination. Treatment is surgical being possible to use conservative or radical techniques. For total or parcial resection, the patient's rehabilitation should be planned returning function and aesthetics to the person. Autogenous bone is considered the "gold standard" when opting for grafting in this kind of injury. The objective of this study is to describe two cases of the treatment consisted in partial mandibulectomy and grafting autogenous non vascularizated iliac crest bone comparing the evolution for a period of 1 year after all interventions performed ate Hospital Municipal Salgado Filho (HMSF) Rio de
\end{abstract}


Janeiro/RJ, Brazil. It could be seen that although the both patient has received the same treatment, the final results was different between two patients.

Key words: Ameloblastoma, Odontogenic Tumor, Autogenous Graft. 


\section{INTRODUÇÃO}

O Ameloblastoma é uma neoplasia odontogênica benigna, com perfil de crescimento lento, infiltrativo e altamente agressivo. Muitas vezes, o paciente evolui clinicamente de forma assintomática. Acredita-se que o tumor se origine dos restos da lâmina dentária, de um órgão do esmalte em desenvolvimento, do revestimento epitelial de um cisto odontogênico, ou das células basais da mucosa oral. ${ }^{(2)}$ É consenso na literatura que a região posterior da mandíbula é a mais afetada, havendo estudos que apontam este osso como sede de $80 \%$ dos diagnósticos de ameloblastoma, sem predileção por gênero ${ }^{(5)}$. A Organização Mundial da Saúde (OMS) definiu em 2017 a classificação das lesões em: Ameloblastoma, Ameloblastoma Unicistico e Ameloblastoma Periférico.

O ameloblastoma unicístico é mais comum em pessoas jovens, entre a primeira e terceira décadas de vida, embora seja raro. Frequentemente surge na região posterior da mandíbula envolvendo a coroa de dentes impactados. Radiograficamente se mostra unilocular e com bordas bem delimitadas, podendo gerar reabsorções radiculares. O tipo multicístico cresce em diversas direções invadindo o tecido local e gerando destruições ósseas de maiores proporções. Nota-se com certa frequência uma expansão das corticais ósseas. O aspecto radiográfico é multilocular, comparado a bolhas de sabão ou favos de mel, além disso, é frequente que haja raízes de molares inferiores envolvidas na lesão radiolúcida. ${ }^{(2)} \mathrm{O}$ ameloblastoma periférico, localiza-se principalmente em gengiva e mucosa alveolar se assemelhando a nódulos sésseis, indolores e normocrômicos. ${ }^{(2)}$ Segundo, Neville, há ainda a variante maligna, muito rara, exibindo lesões agressivas, com margens mal definidas e destruição cortical.

O diagnóstico precoce da lesão é dificultado pelo seu perfil silencioso. Quando os sintomas surgem é comum que o tumor já esteja em uma fase mais avançada. Visando o diagnóstico correto, o exame clínico é indispensável, contudo, não exclui a necessidade de métodos auxiliares como exames de imagem e biópsia incisional. As imagens são fundamentais para verificar a localização, extensão, limites e correlação da lesão com estruturas adjacentes. ${ }^{(2)}$ Além disso a biópsia incisional seguida da análise histopatológica fornece o resultado final com detalhes sobre o aspecto histológico do tumor. ${ }^{(2)} \mathrm{O}$ tratamento de escolha para o ameloblastoma é o cirúrgico, podendo-se utilizar técnicas conservadoras ou radicais a depender do tipo de tumor e de sua apresentação clínica, tais como: abrangência, relação com a anatomia local, idade do paciente e experiência do 
profissional, dentre outros. ${ }^{(2)}$ Quanto as abordagens conservadoras, pode-se citar: a enucleação e a curetagem, com ou sem marsupialização prévia, criocirurgia, eletrocauterização e radioterapia nos casos inoperáveis, enquanto que as ressecções totais ou parciais são classificadas como abordagens radicais. Em seu artigo, Singh M et al. categorizam as lesões em três grupos: menor que $5 \mathrm{~cm}$, entre 5 a $13 \mathrm{~cm}$ e maiores que 13 $\mathrm{cm}$. Para as lesões entre 5 a $13 \mathrm{~cm}$ com envolvimento de tecidos moles e/ou processo coronóide e base de crânio, o tratamento proposto é a ressecção segmentar, ${ }^{(3)}$ sendo orientado manter uma margem de segurança de $1-1,5 \mathrm{~cm}^{(14)}$

Defeitos ósseos na maxila ou mandíbula, ocasionados por traumas, patologias ou outras condições, podem ser corrigidos por meio de técnicas de enxertia. ${ }^{(4)}$ Não há enxerto plenamente ideal, entretanto, o osso autógeno é considerado o "padrão ouro" por reunir a maior parte dos requisitos citados na literatura. ${ }^{(5)}$

O enxerto autógeno pode ser classificado como vascularizado ou não vascularizado. ${ }^{(4)}$ Enxertos não vascularizados são melhor indicados para defeitos pequenos e onde exista uma disponibilidade adequada de tecido mole, idealmente a extensão varia de 5 a $6 \mathrm{~cm}$ de descontinuidade. ${ }^{(4)}$ A literatura recomenda extremo cuidado no uso desses enxertos para defeitos maiores que $9 \mathrm{~cm}$, indicando falha de $17 \%$ para defeitos a partir de $6 \mathrm{~cm}$ e $75 \%$ de falhas em defeitos maiores que $12 \mathrm{~cm}^{(46)}$. Dessa forma, enxertos autógenos podem ser retirados de crista ilíaca, fíbula, escápula, costela ou rádio, preferindo-se os vascularizados para defeitos longos ${ }^{(4)}$. O objetivo deste trabalho é descrever dois casos cujo tratamento consistiu em mandibulectomia parcial e enxertia de osso autógeno de crista ilíaca não vascularizado, comparando as evoluções por um período de um ano após as intervenções cirúrgicas.

\section{RELATO DE CASO}

CASO 1: Paciente E.S.P.S, sexo feminino, melanoderma,48 anos, buscou o serviço de Cirurgia e Traumatologia Buco-Maxilo-Facial do HMSF, apresentando ao exame clínico edentulismo bilateral em região de molares na mandíbula, e discreto aumento de volume, acompanhado de abscesso em região anterior de mandíbula do lado esquerdo relacionado aos elementos 33 e 34, sítio em que foi realizada a biópsia incisional. A análise histopatológica e emissão do laudo final foram realizados pelo Laboratório de Biotecnologia Aplicada da Universidade Federal Fluminense (LABA- UFF, Niterói, RJ, 
Brasil) com o diagnóstico de Ameloblastoma Sólido. Por meio da imagem tomográfica foi possível obter a exata localização e extensão (comprimento mésio-distal de aproximadamente $20 \mathrm{~mm}$ ) assim como avaliar a relação entre a lesão e as estruturas anatômicas faciais. O tratamento eleito foi a abordagem cirúrgica radical com $1 \mathrm{~cm}$ de margem de segurança, seguido da reconstrução com enxerto de osso autógeno não vascularizado da crista ilíaca. O procedimento foi realizado sob anestesia geral, e a paciente evoluiu com deiscência de sutura, formação de sequestro ósseo e remodelação óssea considerável.
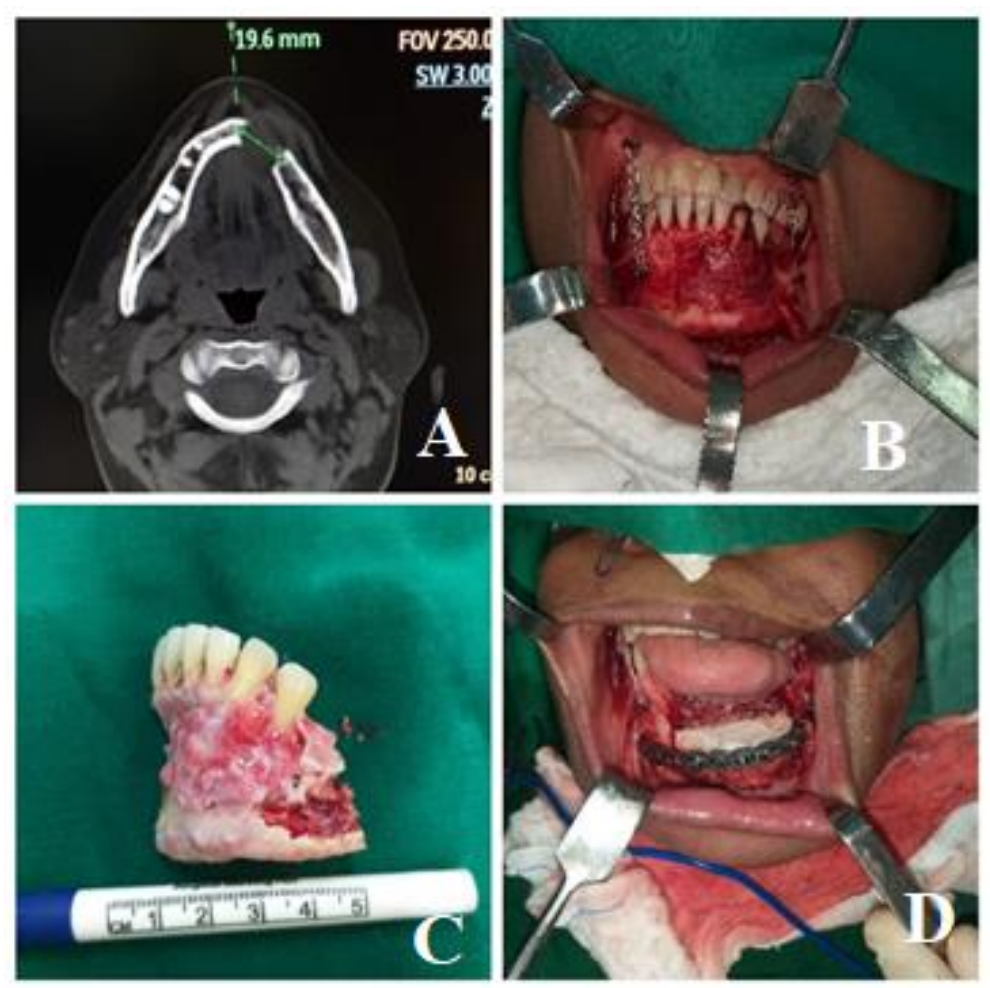

Figura 1: A) Imagem tomográfica corte axial demonstrando extensão da lesão. B) Bloqueio Maxilo-mandibular trans cirúrgico para estabilização de oclusão pré ressecção parcial de mandíbula. C) Fragmento contendo lesão + margens de segurança. D) Enxerto autógeno não vascularizado proveniente de crista ilíaca posicionado e fixado com placa de titânio sistema 2.4 .

CASO 2: Paciente B.O.D, leucoderma, sexo feminino, 23 anos, foi encaminhada ao HMSF para a avaliação de uma pequena fístula com drenagem ativa e espontânea de secreção purulenta em região de $1^{\circ}$ molar inferior esquerdo. Na radiografia panorâmica trazida pela paciente, pôde-se observar uma área radiolúcida, multilocular próxima ao ápice do elemento 35 e envolvendo a raiz mesial do elemento 36. Foi realizada a biópsia 
incisional que possibilitou o diagnóstico de Ameloblastoma. As imagens tomográficas indicaram uma lesão de aproximadamente $25.4 \mathrm{~mm}$. O tratamento proposto foi a mandibulectomia parcial com margens de segurança seguido da reconstrução com enxerto autógeno não vascularizado de crista ilíaca, usando-se parafusos e placa de titânio do sistema 2.4. O procedimento foi realizado sob anestesia geral, e a paciente evoluiu com formação de pequeno sequestro ósseo e moderada remodelação óssea.
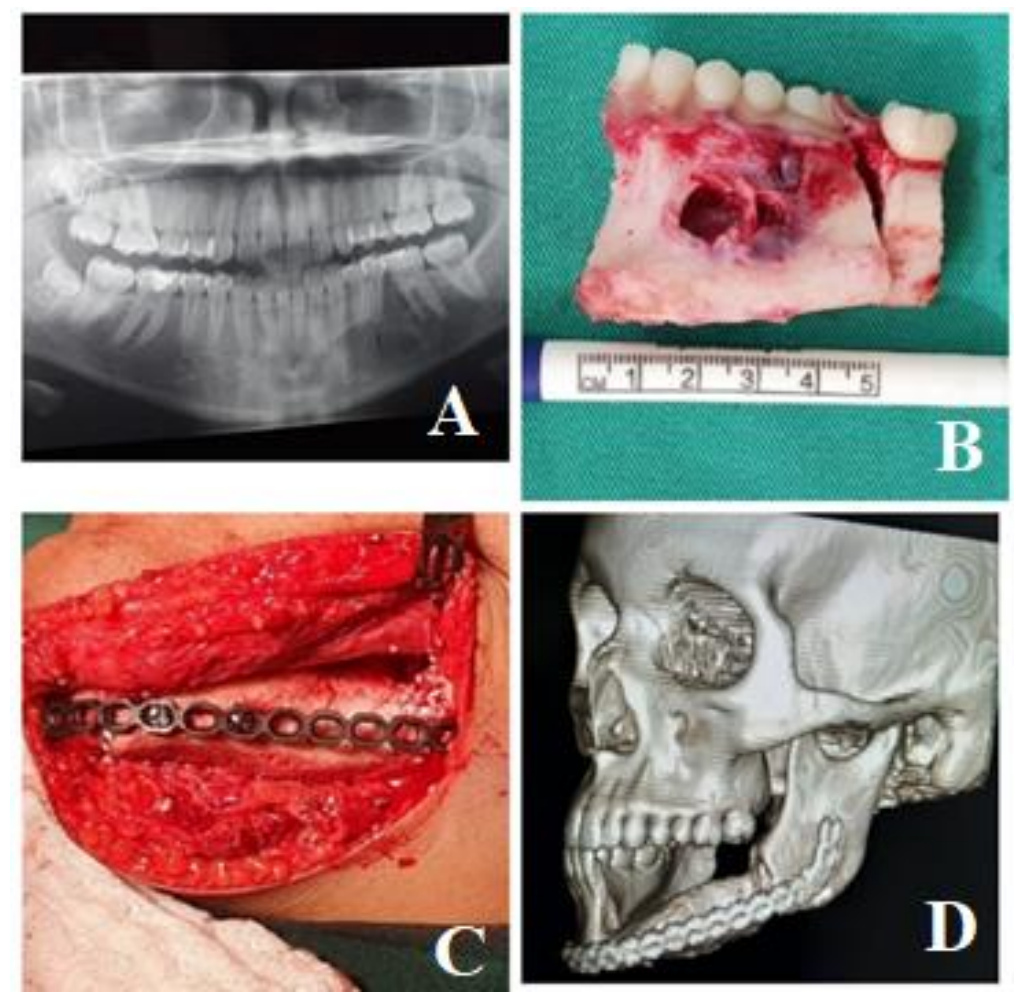

Figura 2: A) Radiografia Panorâmica evidenciando lesão de aspecto multilocular em lado esquerdo da mandíbula, próxima ao ápice do elemento 35 e envolvendo raiz mesial do 36. B) Fragmento ressecado com lesão e margem de segurança. C) Enxerto autógeno não vascularizado de crista ilíaca posicionado e fixado com placa de titânio sistema 2.4 D) Reconstrução 3D tomográfica pós operatória. 


\section{DISCUSSÃO}

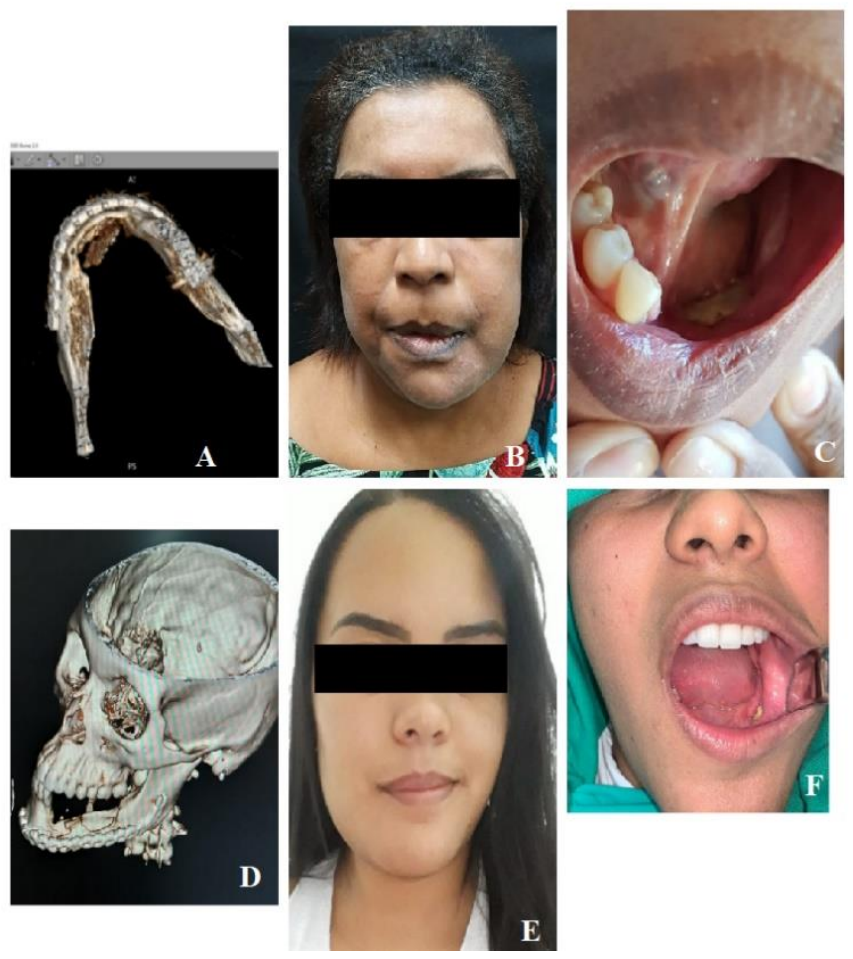

Figura 3: CASO 1 :A) Reconstrução 3D evidenciando sequestro e remodelação óssea após 1 ano de acompanhamento B) Aspecto facial da paciente após 1 ano de acompanhamento C) Exposição intraoral de sequestro ósseo. CASO 2: D) Reconstrução 3D evidenciando remodelação óssea paciente após 1 ano de acompanhamento. E) Aspecto facial da paciente após 1 ano de acompanhamento. F) Exposição intraoral de sequestro ósseo.

Os requisitos para a escolha de um enxerto são: capacidade de osteogênese, osteoindução, osteocondução, ser rapidamente revascularizado, ter fornecimento ilimitado sem comprometer a área doadora, não induzir resposta do sistema imunológico e promover estabilidade e suporte satisfatórios. ${ }^{(5)} \mathrm{O}$ osso autógeno é o padrão ouro em reconstruções ósseas. ${ }^{(5)} \mathrm{Na}$ escolha do osso autógeno, devem ser considerados alguns aspectos, tais como: sítio doador (intra ou extra oral), avaliação correta da quantidade e qualidade de osso disponível para ser doado, a extensão e tipo do defeito ósseo, a morbidade pós cirúrgica, tempo cirúrgico, risco de lesões vasculonervosas, e a capacitação da equipe que realizará o procedimento. ${ }^{(5)}$ Em reconstruções motivadas por ressecção de ameloblastomas, a área doadora deve ser extra oral, uma vez que as mandibulectomias produzem defeitos grandes. Assim, os enxertos são comumente retirados da crista ilíaca, fíbula, calvária, costelas, rádio, tíbia ou escápula. ${ }^{(5)}$. 
Independente do enxerto ser vascular ou avascular, a reconstrução óssea após a ressecção mandibular, tem diversas vantagens: estabilização da fratura, diminuição do risco de fratura da placa, manutenção do contorno facial, e criação de uma área para reabilitação dentária. Se a reconstrução for imediata, reduz-se número de intervenções cirúrgicas e além de proporcionar a reabilitação e o retorno à vida social mais rapidamente. (2 4) Enxertos não vascularizados são melhor indicados e com maiores taxas de sucesso para defeitos pequenos (entre 5-6 cm) e onde haja uma disponibilidade de tecido mole adequada, embora estudos recentes apresentaram casos em que obtiveram sucesso usando enxertos avasculares para defeitos maiores. ${ }^{(467)}$ Enquanto que para os defeitos longos, com infecções extensas, que já sofreram radiações, ou que não possuam tecido mole suficiente, indica-se o enxerto vascularizado. ${ }^{(4)}$

Enxertos não vascularizados de crista ilíaca, quando utilizados dentro da sua indicação, apresentam algumas vantagens: oferece melhor contorno facial no pós cirúrgico quando comparados à fíbula, melhor volume e altura óssea em todas as situações clínicas, maior disponibilidade óssea para posterior reabilitação com implantes, cicatrizes menos visíveis, não necessita de equipamentos específicos e cirurgiões especializados, e por fim um estudo de 2006 demonstrou que a distribuição do estresse mecânico sobre o osso de crista ilíaca foi semelhante à da mandíbula. ${ }^{(6)}$ As limitações se resumem em: morbidade pós operatória, dificuldade de deambular, maior taxa de reabsorção óssea do enxerto devido a sua origem endocondral e um tempo maior de espera até que seja seguro instalar implantes osseointegráveis. ${ }^{(67)}$ Dessa forma, o osso não vascularizado de crista ilíaca tornou-se uma boa opção terapêutica para os casos, uma vez que, do ponto de vista da indicação, ambas as descontinuidades ósseas estavam entre 5-6cm, extensão com maior índice de sucesso reportado pela literatura. Além disso, no HMSF não há uma equipe de Ortopedia especializada e treinada para a remoção de enxertos vascularizados, assim, entende-se que se os defeitos ósseos fossem categorizados como extensos as pacientes precisariam de outra equipe ou outra unidade hospitalar. Por fim, quanto à escolha da crista ilíaca, se deu pelo fato da equipe de CTMBF ter avaliado que dentre os possíveis sítios doadores, como fíbula e costela por exemplo, a crista ilíaca seria o ideal para mimetizar ao máximo o contorno original da mandíbula, além de oferecer maior volume e altura óssea, considerando um grau de remodelação já previsto. 
As complicações mais frequentes são: maior susceptibilidade a infecção com menor taxa de sobrevivência do enxerto, deiscência de sutura com exposição e/ou fratura da placa. ${ }^{(4}$ 7) Alguns autores usam como parâmetro de sucesso evidencias radiográficas de continuidade e estabilidade ósseas em no mínimo 4 meses após a cirurgia, bem como fechamento completo de feridas intra e extra orais. ${ }^{(6)}$ É importante destacar que fatores como idade do paciente, comorbidades, etiologia do defeito, extensão e tipo de enxerto, local do defeito e uso ou não de proteína morfogenética óssea (BMP) são fatores que influenciam nos resultados e devem ser coletados e analisados. ${ }^{(6)}$ Diante disso, nota-se que o tratamento proposto para os casos apresentados, se respalda em todos os aspectos, por dados bem estabelecidos pela literatura, corroborando assim para a confirmação de que fatores diversos e individuais influenciam no resultado final dos casos. Nesse caso pôde-se observar que embora as pacientes tivessem características distintas como idade e condições prévias de saúde, ambas obtiveram sucesso no tratamento proposto. Atualmente uma alternativa aos enxertos é o uso de próteses customizadas. O uso de prototipagem tem se tornado cada vez mais frequente, reduzindo riscos e tempo cirúrgico. (8) A customização utiliza materiais como: hidroxiapatita, silicone, Polimetilmetacrilato (PMMA) e Polietileno Poroso, sendo auxiliada pela prototipagem rápida de um modelo $3 \mathrm{D}$ obtido do próprio paciente.

\section{CONSIDERAÇÕES FINAIS}

O ameloblastoma é um tumor benigno agressivo, resultando em mutilações expressivas quando se opta pelo tratamento cirúrgico radical. O planejamento deve sempre considerar a reconstrução e a reabilitação do paciente, devolvendo estética e função. Quanto às possibilidades de enxertia, o osso autógeno ainda é a melhor opção para a reconstrução de grandes defeitos ósseos. Além disso, este é mais acessível para essas reconstruções no que tange a relação custo-benefício, financeiro e biológico, quando comparado aos enxertos vascularizados e às próteses customizadas. Nos casos apresentados, ambas as reconstruções foram realizadas com osso autógeno não vascularizado de crista ilíaca, associado ao sistema de fixação interna rígida do sistema 2.4, havendo sucesso apesar dos diferentes graus de remodelação. As pacientes se encontram em acompanhamento 
ambulatorial, aguardando a realização da reabilitação estética e funcional por meio de próteses dentárias.

\section{REFERÊNCIAS BIBLIOGRÁFICAS}

${ }^{1}$ Silva LC, Bezerra Junior GL, Salles PHH, Vasconcellos RB, Ribeiro NRB, Parente JLC. Extenso Ameloblastoma em Mandíbula: Relato de Caso. Rev Odontol. Univ, São Paulo, Set- dez 2017; 29(3): 300-4..

http://publicacoes.unicid.edu.br/index.php/revistadaodontologia/article/view/635

2 Domingues MM, Orsine RA, Martins, MAT, Bussadori,

SK, Fernandes KPS. Ameloblastoma: revisão de literatura. ConScientiae Saúde.2007; 6(2): 269-278. https://www.redalyc.org/pdf/929/92960209.pdf

${ }^{3}$ Singh M, Shah A, Bhattacharya A,Raman R, Ranganatha N, Prakash P. Treatment algorithm for ameloblastoma.Case reports in dentistry.2014; 2014:121032.

https://www.ncbi.nlm.nih.gov/pmc/articles/PMC4274852/pdf/CRID2014-121032.pdf

${ }^{4}$ Chae MP, Smoll NR, Hunter-Smith DJ, Rozen WM. Establishing the natural history and growth rate of ameloblastoma with implications for management: systematic review and meta-analysis.

https://journals.plos.org/plosone/article?id=10.1371/journal.pone.0117241

${ }^{5}$ Kenneth JZ. Bone graft harvesting from distant sites:Concepts and Techniques. Oral Maxillofacial Surg Clin. 2010; 22, 301-316.

https://pubmed.ncbi.nlm.nih.gov/20713264/

${ }^{6}$ Pogrel MA, Podlesh S, Anthony J P , Alexander J. A Comparison of Vascularizedand Nonvascularized Bone Grafts for Reconstruction ofMandibularContinuityDefects J Oral MaxillofacSurg 1997; 55:1200-1206.

https://pubmed.ncbi.nlm.nih.gov/9371107/

${ }^{7}$ Marechek A, AlShare A, Pack S, Demko C, Quereshy FA, Baur D. Nonvascularized Bone Grafts for Reconstruction of Segmental

Mandibular Defects: Is Length of Graft a Factor of Success? J Oral Maxillofac Surg. 2019

https://pubmed.ncbi.nlm.nih.gov/31228424/

8 Siqueira AS, Torres LHS, Diniz JÁ, Rodrigues EDR, Uchoa CP, FilhoVAP, et al. Sequela de cirurgia para reconstrução mandibular após ressecção de ameloblastoma. Arch Health Invest. 2019 8(8):425-429. http://www.archhealthinvestigation.com.br/ArcHI/article/view/4632/pdf 